\title{
Progress and challenges in measles and rubella elimination in the WHO European Region
}

\author{
Siddhartha Sankar Datta ${ }^{a},{ }^{\star}$, Patrick Michael O'Connor ${ }^{a}$, Dragan Jankovic ${ }^{a}$, Mark Muscat ${ }^{a}$, \\ Myriam Corrine Ben Mamou ${ }^{a}$, Simarjit Singh ${ }^{a}$, Theodoros Kaloumenos ${ }^{a}$, Susan Reef ${ }^{b}$, \\ Mark Papania ${ }^{b}$, and Robb Butler ${ }^{\mathrm{a}}$ \\ avaccine-preventable Diseases and Immunization, World Health Organization Regional Office for \\ Europe, Denmark \\ ${ }^{b}$ Global Immunization Division, Centres for Disease Control and Prevention, United States
}

\section{Abstract}

Introduction-Despite availability of safe and cost-effective vaccines to prevent it, measles remains one of the significant causes of death among children under five years of age globally. The World Health Organization (WHO) European Region has seen a drastic decline in measles and rubella cases in recent years, and a few of the once common measles genotypes are no longer detected. Buoyed by this success, all Member States of the Region reconfirmed their commitment in 2010 to eliminating measles and rubella, and made this a central objective of the European Vaccine Action Plan 2015-2020 (EVAP). Nevertheless, sporadic outbreaks continue, recently affecting primarily adolescents and young adults with no vaccination or an incomplete vaccination history. The European Regional Verification Commission for Measles and Rubella Elimination was established in 2011 to evaluate the status of measles and rubella elimination based on documentation submitted annually by each country's national verification committee.

Discussion-Each country's commitment to eliminate measles and rubella is influenced by competing health priorities, and in some cases lack of capacity and resources. All countries need to improve case-base surveillance for both measles and rubella, ensure documentation of each outbreak and strengthen the link between epidemiology and laboratory data. Achieving high coverage with measles- and rubella-containing vaccines will require a multisectoral approach to address the root causes of lower uptake in identified communities including service delivery challenges or vaccine safety concerns caused by circulating myths about vaccination.

Conclusions-The WHO European Region has made steady progress towards eliminating measles and rubella and over half of the countries interrupted endemic transmission of both

\footnotetext{
*Corresponding author at: Vaccine-preventable Diseases and Immunization, World Health Organization Regional Office for Europe, UN City, Marmorvej 51, DK-2100 Copenhagen Ø, Denmark. dattas@who.int (S.S. Datta).

Contributions by authors

Siddhartha Sankar Datta and Patrick O'Connor designed the draft of the manuscript. Simarjit Singh and Theodoros Kaloumenos supported the data analysis and preparation of the figures for the manuscript. Myriam Ben Mamou reviewed the laboratory sections of the manuscript and developed the figure on the measles genotype distribution. Dragan Jankovic, Mark Muscat, Susan Reef, Mark Papania and Robb Butler provided their critical reviews to the intellectual content of the paper. All authors have approved the final version that was submitted for publication.

Conflicts of interest

None.
} 
diseases by 2015 . The programmatic challenges in disease surveillance, vaccination service delivery and communication in the remaining endemic countries should be addressed through periodic evaluation of the strategies by all stakeholders and exploring additional opportunities to accelerate the ongoing elimination activities.

\section{Keywords}

Disease elimination; European Region; Measles; Rubella; Progress; Verification; Vaccines

\section{Introduction}

Measles and rubella are both highly contagious viral diseases. Worldwide, measles causes significant mortality in children under five years of age [1] with the fatality rates varying from $0.05 \%$ [2] in developed countries to as high as $30 \%$ in emergency settings (e.g. among refugees and disadvantaged populations) [3]. Globally there has been a marked decline in measles and rubella cases and deaths following the widespread use of safe and cost-effective measles- and rubella-containing vaccines in national childhood vaccination programmes. The World Health Organization (WHO) estimates that measles-related deaths have declined globally from about 548,300 in 2000 [4] to an estimated 114,900 in 2014 [5]. The reported rubella cases declined from about 670,800 in 2000 to an estimated 33,100 in 2014, although rubella surveillance data are inconsistent [6].

Elimination of measles, rubella and congenital rubella syndrome (CRS) are goals of the Global Vaccine Action Plan (GVAP) endorsed by the World Health Assembly in 2012 [7]. All WHO regions have now established goals to eliminate measles (and in some regions also rubella) by 2020 [5].

In the European Region, all 53 Member States have committed to elimination of both measles and rubella. In the September 2010 meeting of the WHO European Regional Committee, Member States renewed their commitment to eliminate measles and rubella and prevent CRS by 2015 by, (1) increasing demand for and delivery of vaccination to achieve and sustain $\geq 95 \%$ coverage with two doses of measles-containing vaccine across a wide age range, (2) implementing effective outbreak control measures, and (3) further strengthening surveillance to identify cases and outbreaks promptly, and to validate elimination [8]. In September 2014, all Member States reaffirmed their commitment to the goal of measles and rubella elimination as part of the endorsement of the European Vaccine Action Plan 20152020 (EVAP) by the WHO European Regional Committee [9]. While a number of countries in the Region are still endemic for measles and rubella, a growing number of countries have interrupted endemic transmission of these diseases. This report summarizes the progress made towards measles and rubella elimination in the WHO European Region.

\subsection{Measles and rubella cases}

The number of measles cases in the Region declined from over 185,000 in 1990 to 24,957 in 2015 [10] (see Fig. 1). The number of reported rubella cases declined from 29,617 in 2012 to 2437 in 2015 [10]. Data submitted by countries show that 2535 measles cases were reported in 33 countries while 1090 rubella cases were reported in 17 countries in the 
Region during the first three-quarters of 2016 [WHO surveillance database, data not published]. Compared to the same period in 2015, the Region has seen an $89 \%$ reduction in the number of reported measles cases and an $80 \%$ reduction in the number of reported rubella cases in 2016. In both years fewer countries reported rubella cases (including zero reporting) than measles cases $[10,11]$.

Despite significant efforts made by countries to improve the notification of cases, some national surveillance systems in the Region still fail to investigate and report every suspected case of measles or rubella. Therefore no laboratory testing is conducted to confirm the diagnosis of many fever and rash cases. At this stage of elimination, incomplete case investigations remain a concern throughout the Region.

\subsection{Routine and supplementary immunization activities}

All WHO Member States are expected to report routine vaccination coverage data to WHO and the United Nations Children's Fund (UNICEF) in a standardized Joint Reporting Form. All 53 countries in the Region have introduced two doses of measles-rubella vaccination in their national childhood immunization schedules [12], and in 2015, all but two reported their routine vaccination coverage. Analysis of this data showed that 25 of the 51 reporting countries achieved the WHO-recommended coverage of greater than $95 \%$ for the first dose of measles-containing vaccine (MCV1) and 15 countries achieved this target for the second dose of measles-containing vaccine (MCV2) [13].

In 2015, five countries (Azerbaijan, Cyprus, Georgia, Kazakhstan, Kyrgyzstan) conducted different varieties of supplementary immunization activities (SIAs) covering various age groups using measles (M), measles-rubella (MR) or measles-mumps-rubella (MMR) vaccine. Azerbaijan, Cyprus and Kyrgyzstan have attained coverage of more than $85 \%$ in these activities. Kyrgyzstan reported more than $90 \%$ in both SIAs conducted [13] (see Figs. 2 and 3$)$.

\subsection{Characteristics of recent outbreaks}

Widespread outbreaks of measles and rubella have been reported by just a few countries in the Region in the recent years. Large-scale measles outbreaks in 2015 were reported by Bosnia and Herzegovina, Germany, Kyrgyzstan and the Russian Federation [10]. Over half of the laboratory-confirmed measles cases in 2015 were predominantly among adolescents and young adults while $20 \%$ were among infants less than 12 months of age. Only $15 \%$ had received two doses of measles vaccine, $29 \%$ were not immunized, $11 \%$ were incompletely immunized and 45\% had an unknown immunization status. Rubella outbreaks in 2015 were reported by Georgia, Kyrgyzstan, Poland and Ukraine [10,14], with the majority of cases reported among children less than 15 years of age. The countries' immunization response following the measles-outbreaks varied from raising awareness among health physicians and parents to post-exposure vaccination and conducting catch-up vaccinations in the affected areas [14] (see Figs. 4 and 5). 


\subsection{Vulnerable population groups affected}

Some countries reporting both sporadic and widespread measles cases in recent years have also reported high national measles vaccination coverage. A considerable proportion of these cases had not been fully vaccinated. Despite reportedly high vaccination coverage at national level, pockets of sub-optimal vaccination coverage in the Region persist. Since 2010, outbreaks have been reported in particular population groups in the region, where there is a lower probability of being vaccinated: Roma communities in Bulgaria, Anthroposophists in Germany, the Netherlands and Switzerland, Jewish ultra-orthodox communities in Belgium, Israel, United Kingdom and orthodox Protestants in the Netherlands [15-17]. However, large outbreaks reported in France (2011), United Kingdom (2014) and Germany (2015) revealed the vulnerability of certain age groups in the general population that had not benefited from vaccination due to varied reasons.

Furthermore, susceptible health care workers (HCWs) $[14,17]$ played an important role in nosocomial infection in a few of the measles outbreaks in the Region. Approximately 68 HCWs in the Czech Republic, 13 HCWs in Latvia and 30 in Spain were affected in 2014 and 9 were affected in Croatia in 2015 [14,17]. Most of the affected HCWs were either unvaccinated or their vaccination status was unknown. Most countries in Europe do not have a measles vaccination requirement for $\mathrm{HCWs}$ and even those which recommend such, the policies are variably implemented [18].

\subsection{Surveillance activities}

Over the past few years, countries have undertaken several steps to strengthen both the epidemiological and laboratory components of their surveillance system but the quality of the existing surveillance varies considerably across the Region.

The WHO Regional Office for Europe (Regional Office) receives monthly reports of measles and rubella cases from Member States, which include case-based and aggregate reporting with both clinical and laboratory confirmation of the cases. Rubella and CRS surveillance, however, remain a challenge with only a few countries having an established system for monitoring and reporting.

\subsection{Measles and rubella laboratory activities}

A critical component of laboratory surveillance is the genetic characterization of wild-type measles and rubella viruses [19]. The genotyping data of the detected viruses provide evidence to document the interruption of endemic transmission of measles and rubella. However, the genotype reports alone do not sufficiently indicate the global or regional distribution of the viruses, as there is a strong bias in reporting of the data to the sequence database for measles and rubella [19]. Genetic data of the detected viruses should be combined with case-based epidemiological data to determine the transmission pathways of the circulating virus chains [20].

The measles and rubella laboratories in the European Region are part of the global WHO measles and rubella laboratory network, which provides confirmation of suspected cases of measles and rubella including genetic characterization of the circulating strains of identified 
wild-viruses. All (71) measles and rubella reference laboratories in the Region have retained their annual accreditation status through to 2015 .

Molecular genotyping of the viruses has become increasingly important at this stage of the elimination process in European Region. Most countries with confirmed measles cases have submitted genomic sequence information to the measles nucleotide surveillance database; however, only a few countries with confirmed rubella cases have submitted genomic sequence information on rubella cases to the rubella nucleotide surveillance database [21]. Analysis of the available genotypic information indicates that a few of the measles genotypes are no longer detected in the European Region, thereby suggesting that several long-lasting transmission chains have been interrupted. This underlines the progress made by the Region towards measles elimination (see Fig. 6). Measles genotypes C2 and D7 have not been detected since 2004, and genotype D6 and a subvariant of D4 (Bucharest. ROU/ 48.04) have not been detected since 2007 [22]. Since 2012, genotypes B2, B3, D4, D8, D9, G3 and H1 have been detected in the European Region, of which B3, D4 and D8 have been predominant and the others have been detected sporadically in a few countries [22]. The measles genotypes identified in 2015 were B3, D4, D8, D9, and H1 [23]. The predominant measles genotypes reported in 2015 included several lineages of D8 reported by 27 countries and the variants of measles genotype B3 reported by 16 countries [14]. The major rubella genotypes identified in 2015 were $2 \mathrm{~B}, 1 \mathrm{E}$ and 1a [24].

\subsection{Measles and rubella verification activities}

The European Regional Verification Commission for Measles and Rubella Elimination (RVC), an independent panel of experts, was established in 2011 [25] to evaluate the status of measles and rubella elimination in the Region based on the September 2010 resolution by the WHO Regional Committee for Europe. The vaccine-preventable diseases and immunization programme (VPI) of the Regional Office serves as the secretariat of the RVC.

The verification process involves examination of the evidence presented by each country's national verification committee (NVC) based on the WHO-recommended performance indicators for both surveillance and immunization coverage [26]. As part of the verification process in the Region, each country submits documentation to RVC every year; a process which will continue for at least three years after the RVC confirms the interruption of endemic measles and rubella transmission in all Member States of the Region. The evaluation of the countries by the RVC according to the established criteria outlined verification framework for measles and rubella elimination in the WHO European Region is evidence-based, measurable and independent.

The RVC reviews information on measles and rubella epidemiology, molecular data, population immunity, and surveillance and classifies the countries according to WHO's definition of elimination, i.e., the absence of endemic measles or rubella cases in a geographic area for a period of at least 12 months or more in the presence of a wellperforming surveillance system [26]. Elimination can be verified once a country has sustained interruption of endemic transmission for 36 consecutive months. According to the verification framework for measles and rubella elimination in the Region, the endemic measles or rubella transmission will be considered reestablished when the epidemiological 
and laboratory evidence indicate that a chain of transmission of a virus variant has continued uninterrupted for a period of 12 months or more in a defined geographical area where the disease was previously eliminated [27]. By the end of 2015, none of the countries of the Region were found to have reestablished endemic measles or rubella transmission. The review of the measles/rubella elimination status in other WHO Regions shows that two countries namely Brazil in WHO Region of Americas and Mongolia in WHO Western Pacific Region were verified to have reestablished endemic measles transmission by their respective regional verification commissions in recent past [28,29].

In 2014, the verification procedures were modified by the RVC to allow verification of elimination at the national level as opposed to only at the regional level [30]. Similarly, the RVC revised the Annual Status Update reports (ASU) to include information on effective linkages between laboratory and epidemiological surveillance as well as documentation on chains of transmission [30].

The September 2010 resolution also urged each Member State to establish an NVC [8]. As of December 2016, 51 out of 53 countries have functioning NVCs [31], including the Scandinavian Verification Committee established by Denmark, Norway and Sweden. Monaco and San Marino have not established NVCs.

Following its review of the 2015 reports submitted by the NVCs, the RVC concluded that by 2015, 24 countries had eliminated measles and another 13 had interrupted endemic measles transmission; 24 had eliminated rubella and another 11 had interrupted endemic rubella transmission [31].

\subsection{Support to the measles endemic countries by the WHO secretariat}

Recognizing the need for accelerated actions by countries and scaled-up support from WHO and other partners towards achieving the Region's measles and rubella elimination target, the Regional Office introduced a package of accelerated actions in 2013 [32] to step up the support in high-priority countries. The RVC members and Regional Office staff conducted advocacy and technical missions to these countries [21], where country-specific issues were identified and the necessary technical support was provided. In 2014 and 2015, teams visited Austria, Bulgaria, Croatia, Cyprus, France, Greece, Italy, the Russian Federation and Spain; and in 2015, outbreak support was provided to Bosnia and Herzegovina, Kazakhstan and Kyrgyzstan [21]. In August 2016, WHO conducted a technical consultation with the United States Centers for Disease Control and Prevention (CDC) and European Centre of Disease Prevention and Control (ECDC) to review the situation of measles and rubella in endemic countries of the Region and identify priority elimination activities in these countries. A draft global measles-rubella categorization method [33] was used to identify specific determinants (immunization status and surveillance performance). This continuum exercise was planned to further enhance the support to the identified priority countries in the WHO European region. Country profiles outlining the challenges, interventions and priority activities are being developed following this consultation and will be shared with countries to scale up support and accelerate the elimination efforts in each of these measles and rubella endemic countries. 


\section{Discussion}

The commitment by the Member States of the WHO European Region to the goal of eliminating measles and rubella is signaled by the renewed commitment to elimination of measles and rubella and prevention of CRS by 2015 in 2010 [8] and as part of the reaffirmed commitment in 2014 [9] both of which are in line with the September 2005 resolution to strengthen the national immunization systems through measles and rubella elimination and prevention of congenital rubella infection [34]. Despite the high-level commitments made by countries in the WHO European Region, competing health priorities and the lack of capacity and resources in some countries jeopardize the ability of the Region as a whole to achieve measles and rubella elimination in the near future.

The RVC decision to shift the verification process from the regional level to the country level provides WHO and partners the opportunity to recognize individual country achievements and customize interventions based on a country's size, health structure or surveillance system.

The presence of a strong national surveillance system is essential to detect all cases of measles and rubella, conduct detailed case investigation, laboratory confirm suspected cases and collect genotypic information on the chains of transmission. Implementation of standardized case-based measles and rubella surveillance across the Region is a particular challenge. While case-based measles surveillance is conducted in most of the countries in the Region, there is no comprehensive rubella surveillance system in Belgium, Denmark and France [20]. However, there is progress in Denmark where rubella is expected to become a notifiable condition in 2017 [WHO internal communication]. As more countries are verified as having eliminated measles and rubella, it is critical to improve case-base surveillance of both measles and rubella with emphasis on strengthening the link between epidemiology and laboratory data. At this stage of the elimination process, documenting every outbreak supported by genetic data is crucial to distinguish remaining endemic chains of transmission from importations. The Member States have been providing these critical information in the ASUs; however, it is not sufficient, and particular attention should be paid to rubella and CRS surveillance supported by genetic sequencing data.

The verification framework for measles and rubella elimination in the WHO European Region recommends achieving and sustaining greater than 95\% coverage for the first and second doses of measles- and rubella-containing vaccines at subnational administrative levels $[27,35]$. The officially reported national coverage with the first and second doses of measles- and rubella-containing vaccine by the Member States shows a wide variation from $53 \%$ to $99 \%$ [13]. Equally wide variations in the coverage level are witnessed at the subnational levels. The region-specific information provided by the Member States in the 2015 WHO/UNICEF Joint Reporting Form indicate that most of the countries in the region either do not require a proof or no information is available on mandatory check for vaccination at school entry. In line with the guidance provided in the WHO measles position paper [36] on screening of children for their measles vaccination history at the time of school entry and those lacking evidence of receipt of 2 doses should be vaccinated, this is considered a lost opportunity. 
The EVAP envisions a Region in which every person has equitable access to high-quality, safe and affordable vaccines and immunization services throughout the life-course [37]. Elimination of both measles and rubella is one of the six primary goals of the EVAP. Attaining and sustaining high vaccination coverage with the first and second doses of measles- and rubella-containing vaccines would also require efforts to address identified immunity gaps. Every opportunity should be used by countries to ensure that all children in the Region are protected by the recommended two doses of measles- and rubella-containing vaccine.

As adolescents and adults have also been affected in significant proportions throughout the Region, countries need to explore innovative ways to effectively reach these populations. The immunity gaps also comprise individuals with vaccine hesitancy and anti-vaccination sentiment. A multisectoral, comprehensive approach in identifying and addressing underand unimmunized communities or subgroups throughout the Region is needed.

\section{Conclusions}

The Member States of the WHO European Region have made steady progress towards eliminating measles and rubella with over half having interrupted endemic transmission of both diseases by the end of 2015. However, as a whole, the Region faces several challenges in immunization coverage and surveillance performance. This is a highly diverse Region that includes countries with widely varying population sizes and languages, and with complex public health systems that demand extensive coordination mechanisms.

To achieve and sustain regional elimination, it will be crucial for all countries to invest in ways to identify and target vulnerable and high-risk populations and subgroups including HCWs through country-specific tailored strategies to close the existing immunity gaps. The strategies should involve strengthening routine immunization and conducting targeted supplementary immunization activities. In addition, evidence-based, tailored communication initiatives should be developed to raise awareness and address any vaccine safety concerns. The annual European Immunization Week is an excellent opportunity for national programmes to raise awareness among various stakeholders, including parents of unimmunized children. It will also be crucial for all countries to have a highly sensitive surveillance system in place with the ability to identify cases, supported by the accredited laboratory network and respond quickly and adequately to outbreaks. Improvements in measles surveillance in the Region should be capitalized upon to improve rubella surveillance, including reporting of genomic sequence data.

WHO and other development partners along with Member States should periodically evaluate the current elimination activities and explore opportunities to accelerate action and overcome the identified challenges. Inadequate vaccination coverage and persistent occurrence of measles and rubella cases point to critical programmatic issues which should be systematically addressed. Best practices implemented in countries that have eliminated the diseases must be maintained while the remaining countries take action to ensure that they, and thereby also the Region as a whole, can achieve elimination status in the nearest possible future. 


\section{Acknowledgements}

The authors thank all the countries in the WHO European Region for providing the epidemiological and laboratory surveillance data on measles and rubella. The authors also extend their gratitude to Catharina de Kat for her suggestions in the preparation of this article.

Funding

None.

\section{References}

[1]. Black RE, Cousens S, Johnson HL, Lawn JE, Rudan I, Bassani DG, et al. Global, regional, and national causes of child mortality in 2008: a systematic analysis. Lancet 2010;375:1969-87. http://dx.doi.org/10.1016/S0140-6736(10)605491 . Available at [http://dx.doi.org/10.1016/ S0140-6736(10)605491http://www.sciencedirect.com/science/article/pii/S0140673610605491. Available at [ http://www.sciencedirect.com/science/article/pii/S0140673610605491]. [PubMed: 20466419]

[2]. Wolfson LJ, Grais RF, Luquero FJ, Birmingham ME, Strebel PM. Estimation of measles case fatality ratios: a community-based studies. Int J Epidemiol 2009;38:192-202. http://dx.doi.org/ 10.1093/ije/dyn224 . Available at [http://dx.doi.org/10.1093/ije/dyn224http:// ije.oxfordjournals.org/content/38/1/192.full.pdf+html. Available at [ http://ije.oxfordjournals.org/ content/38/1/192.full.pdf+html]. [PubMed: 19188207]

[3]. Shears P, Berry AM, Murphy R, Nabil MA. Epidemiological assessment of the health and nutrition of Ethiopian refugees in emergency camps in Sudan, 1985. Br Med J (Clin Res Ed) 1987;295:314-8. Available at [https:/www.ncbi.nlm.nih.gov/pmc/articles/PMC1247152/pdf/ bmjcred00031-0034.pdf].

[4]. World Health Organization. Progress in global control and regional elimination of measles, 20002011. Wkly Epidemiol Rec 2013;88:29-36. Available at [http://www.who.int/wer/2013/ wer8803.pdf]. [PubMed: 23393700]

[5]. World Health Organization Measles fact sheet, reviewed March 2016 Available at [http:// www.who.int/mediacentre/factsheets/fs286/en/].

[6]. CDC. Global progress toward rubella and congenital rubella syndrome control and elimination, 2000-2014. MMWR Morb Mortal Wkly Rep 2015;64:1052-5. Available at [https:// www.cdc.gov/mmwr/preview/mmwrhtml/mm6437a5.htm]. [PubMed: 26401958]

[7]. World Health Organization. Global Vaccine Action Plan. Geneva, Switzerland: World Health Organization; 2012 Available at [http://apps.who.int/iris/bitstream/ 10665/78141/1/9789241504980_eng.pdf?ua=1].

[8]. World Health Organization Regional Office for Europe. Regional Committee for Europe. Sixtieth session; 2010. Renewed commitment to elimination of measles and rubella and prevention of congenital rubella syndrome by 2015 and sustained support for polio-free status in the WHO European Region. Available at [http://www.euro.who.int/_data/assets/pdf_file/0016/122236/ RC60_eRes12.pdf].

[9]. World Health Organization Regional Office for Europe. Regional Committee for Europe. Sixtyfourth session; 2014. Committee resolution EUR/RC64/R5 on European Vaccine Action Plan 2015-2020. Available from [https://euro.sharefile.com/share?\#/view/s79afa0ede934b2c8].

[10]. World Health Organization [Internet site]. Disease incidence, WHO/UNICEF joint reporting process. Geneva: WHO; 2016 Available from [http://www.who.int/entity/immunization/ monitoring_surveillance/data/incidence_series.xls?ua=1] [accessed on 05 January 2017].

[11]. World Health Organization Regional Office for Europe [Internet site]. Measles and Rubella Data, Centralized information system for infectious diseases. Available from [http://data.euro.who.int/ cisid/] [accessed 05 January 2017].

[12]. World Health Organization [Internet site]. Immunization schedule, WHO/ UNICEF joint reporting process. Geneva: WHO; 2016 Available from [http://apps.who.int/ immunization_monitoring/globalsummary/schedules] [accessed on 05 January 2017]. 
[13]. World Health Organization [Internet site]. Immunization coverage, WHO/ UNICEF joint reporting process. Geneva: WHO; 2016 Available at [http://www.who.int/immunization/ monitoring_surveillance/data/administrative_coverage.xls?ua=1] [accessed on 05 January 2017].

[14]. World Health Organization Regional Office for Europe. WHO EpiBrief, 2016,1: 1-8. Available at [http://www.euro.who.int/_data/assets/pdf_file/0009/313020/EpiBrief_EpiData_1_2016rev1.pdf?ua=1].

[15]. Under-vaccinated groups in Europe: Who are they and how to communicate with them in outbreak situations? Work Package 6 [Internet site]. Effective Communication in Outbreak Management for Europe. Available at [http://ecomeu.info/wp-content/uploads/2015/11/ECOMUnder-vaccinated-groups-in-Europe-WP6.pdf] [accessed on 05 January 2017].

[16]. Muscat M, Marinova L, Mankertz A, Gatcheva N, Mihneva Z, Santibanez S, et al. The measles outbreak in Bulgaria, 2009-2011: An epidemiological assessment and lessons learnt. Euro Surveill. 2016;21(9). http://dx.doi.org/10.2807/1560-7917.ES.2016.21.9.30152.pii=30152 . Available at [http://dx.doi.org/10.2807/1560-7917.ES.2016.21.9.30152.pii=30152http:// www.eurosurveillance.org/ViewArticle.aspx?ArticleId=21399. Available at [ http:// www.eurosurveillance.org/ViewArticle.aspx?ArticleId=21399].

[17]. World Health Organization Regional Office for Europe. WHO EpiBrief, 2014; 2:1-8. Available at [http://www.euro.who.int/_data/assets/pdf_file/0020/254234/EpiBrief_2_2014-rev3-withdata.pdf?ua=1]

[18]. Maltezou HC, Poland GA. Vaccination policies for healthcare workers in Europe. Vaccine 2014;32:4876-80. http://dx.doi.org/10.1016/j.vaccine.2013.10.046 . Available at [http:// dx.doi.org/10.1016/j.vaccine.2013.10.046http://ac.els-cdn.com/S0264410X13014242/1-s2.0S0264410X13014242-main.pdf?_tid=c529d7dcd805-11e6-996b-00000aab0f01\&acdnat=1484143177_c0ddb1c744bcb9903a37f340e86213ff. Available at [ http://ac.els-cdn.com/S0264410X13014242/1-s2.0-S0264410X13014242main.pdf?_tid=c529d7dcd805-11e6-996b-00000aab0f01\&acdnat=1484143177_c0ddb1c744bcb9903a37f340e86213ff]. [PubMed: 24161573]

[19]. World Health Organization. Genetic diversity of wild-type measles viruses and the global measles nucleotide surveillance database (MeaNS). Wkly Epidemiol Rec 2015;90:373-7. Available at [http://www.who.int/wer/2013/wer8803.pdf]. [PubMed: 26211016]

[20]. World Health Organization Regional Office for Europe. WHO EpiData, Oct. 2015 - Sept. 2016, 1-2. Available at [http://www.who.int/wer/2015/wer9030.pdf].

[21]. World Health Organization Regional Office for Europe. Copenhagen 2016. Immunization highlights 2015, report of the WHO Regional Office for Europe. Available at [www.euro.who.int/ __data/assets/pdf_file/0018/307620/Immunization-highlights-2015.pdf?ua=1].

[22]. Mankertz A, Mulders M, Shulga S, Kremer J, Brown K, Santibanez S, et al. Molecular Genotyping and epidemiology of measles virus transmission in the World Health Organization European Region, 2007-2009. J Infect Dis 2011;204:S335-42. http://dx.doi.org/10.1093/infdis/ jir101 . Available at [http://dx.doi.org/10.1093/infdis/jir101http://jid.oxfordjournals.org/ content/204/suppl_1/S335.full.pdf+html. Available at [ http://jid.oxfordjournals.org/content/204/ suppl_1/S335.full.pdf+html]. [PubMed: 21666182]

[23]. Measles Nucleotide Surveillance database (MeaNS). [http://www.who-measles.org]

[24]. Rubella Nucleotide Surveillance database (RubeNS). [http://www.who-rubella.org]

[25]. World Health Organization Regional Office for Europe [Internet site]. Regional verification commission for measles and rubella elimination. Copenhagen, 2016 Available at [http:// www.euro.who.int/en/health-topics/communicable-diseases/measles-and-rubella/activities/ regional-verification-commission-for-measles-and-rubella-elimination-rvc].

[26]. Biellik R, Davidkin I, Esposito S, Lobanov A, Kojouharova M, Pfaff G, et al. Slow progress in finalizing measles and rubella elimination in the European Region. Health Affairs 2016;35:3226. 10.1377/hlthaff.2015.1055. [PubMed: 26858387]

[27]. World Health Organization Regional Office for Europe. Framework for the verification process in the WHO European Region, Copenhagen, Denmark Available at [http://www.euro.who.int/ __data/assets/pdf_file/0009/247356/Eliminating-measles-and-rubella-Framework-for-theverification-process-in-the-WHO-European-Region.pdf]. 
[28]. World Health Organization Regional Office for the Americas. Plan of action for maintaining measles, rubella, and congenital rubella syndrome elimination in the Region of the Americas: Final report. Available at [http://www.paho.org/hq/index.php? option=com_content\&view=article \&id=12276\%3A2016-55th-directing-councildocuments \&catid=8811\%3Adc-documents $\&$ Itemid=42078\&lang=en].

[29]. Patel M, Gacic-Dobo M, Strebel P, Dabbagh A, Mulders M, Okwo-Bele J, et al. Progress toward regional measles elimination - Worldwide, 2000-2015. MMWR Morb Mortal Wkly Rep 2016;65:1228-33. 10.15585/mmwr.mm6544a6. [PubMed: 27832050]

[30]. Meeting report of 4th Meeting of the European Regional Verification Commission for Measles and Rubella Elimination, 26-29 October 2015, Copenhagen, Denmark Available at [http:// www.euro.who.int/_data/assets/pdf_file/0011/304958/4th-RVC-meeting-report.pdf?ua=1].

[31]. Meeting report of 5th Meeting of the European Regional Verification Commission for Measles and Rubella Elimination, 24-26 October 2016, Copenhagen, Denmark Available at [http:// www.euro.who.int/_data/assets/pdf_file/0005/330917/5th-RVC-meeting-report.pdf?ua=1].

[32]. World Health Organization Regional Office for Europe. Package for accelerated action 20132015. Copenhagen: World Health Organization; 2013 Available at [http://www.euro.who.int/ __data/assets/pdf_file/0020/215480/PACKAGE-FOR-ACCELERATEDACTION-20132015.pdf].

[33]. World Health Organization. Meeting of the Strategic Advisory Group of Experts on immunization, October 2016 - conclusions and recommendations. Wkly Epidemiol Rec 2016;91:570-1. Available at [http://apps.who.int/iris/bitstream/10665/251810/1/WER9148.pdf? $\mathrm{ua}=1]$.

[34]. World Health Organization Regional Office for Europe. Regional Committee for Europe resolution EUR/RC55/R7 on strengthening national immunization systems through measles and rubella elimination and prevention of congenital rubella infection in WHO's European Region. Copenhagen: WHO Regional Office for Europe; 2005 Available at [http://www.euro.who.int/ __data/assets/pdf_file/0003/88086/RC55_eres07.pdf?ua=1].

[35]. World Health Organization Regional Office for Europe. Eliminating measles and rubella and preventing congenital rubella infection, Copenhagen, Denmark Available at [http:// www.euro.who.int/_data/assets/pdf_file/0008/79028/E87772.pdf?ua=1].

[36]. World Health Organization. Measles vaccines: WHO position paper - April 2017. Wkly Epidemiol Rec 2017;92:221-2., http://apps.who.int/iris/bitstream/10665/255149/1/ WER9217.pdf?ua=1.

[37]. World Health Organization Regional Office for Europe. European Vaccine Action Plan 20152020. Copenhagen: World Health Organization; 2015 Available at [http://www.euro.who.int/ __data/assets/pdf_file/0007/255679/WHO_EVAP_UK_v30_WEBx.pdf?ua=1]. 


\section{Measles Incidence Rate, WHO European Region}

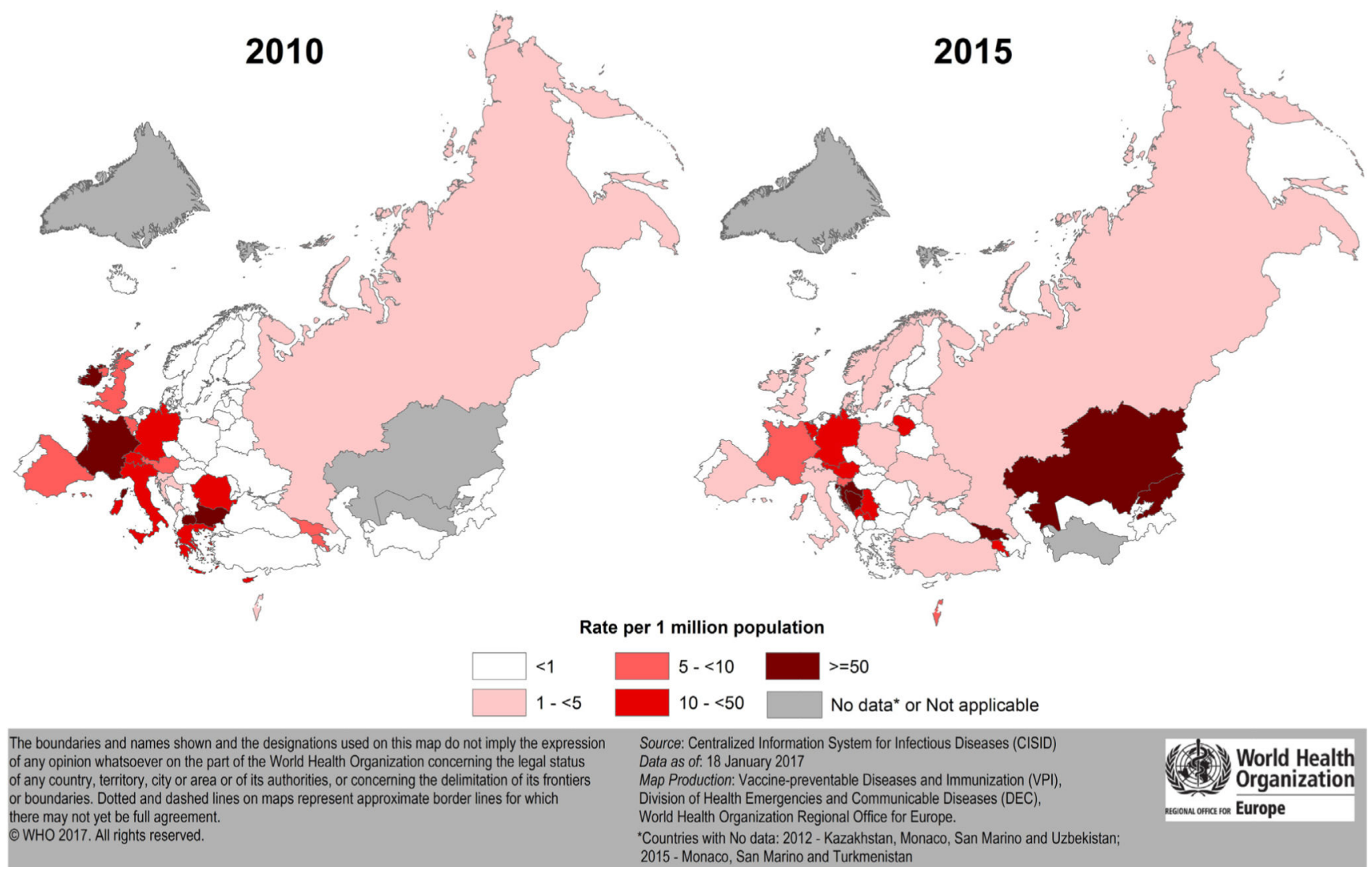

Fig. 1.

Comparison of measles incidence rates in 2010 and 2015 in the WHO European Region. 


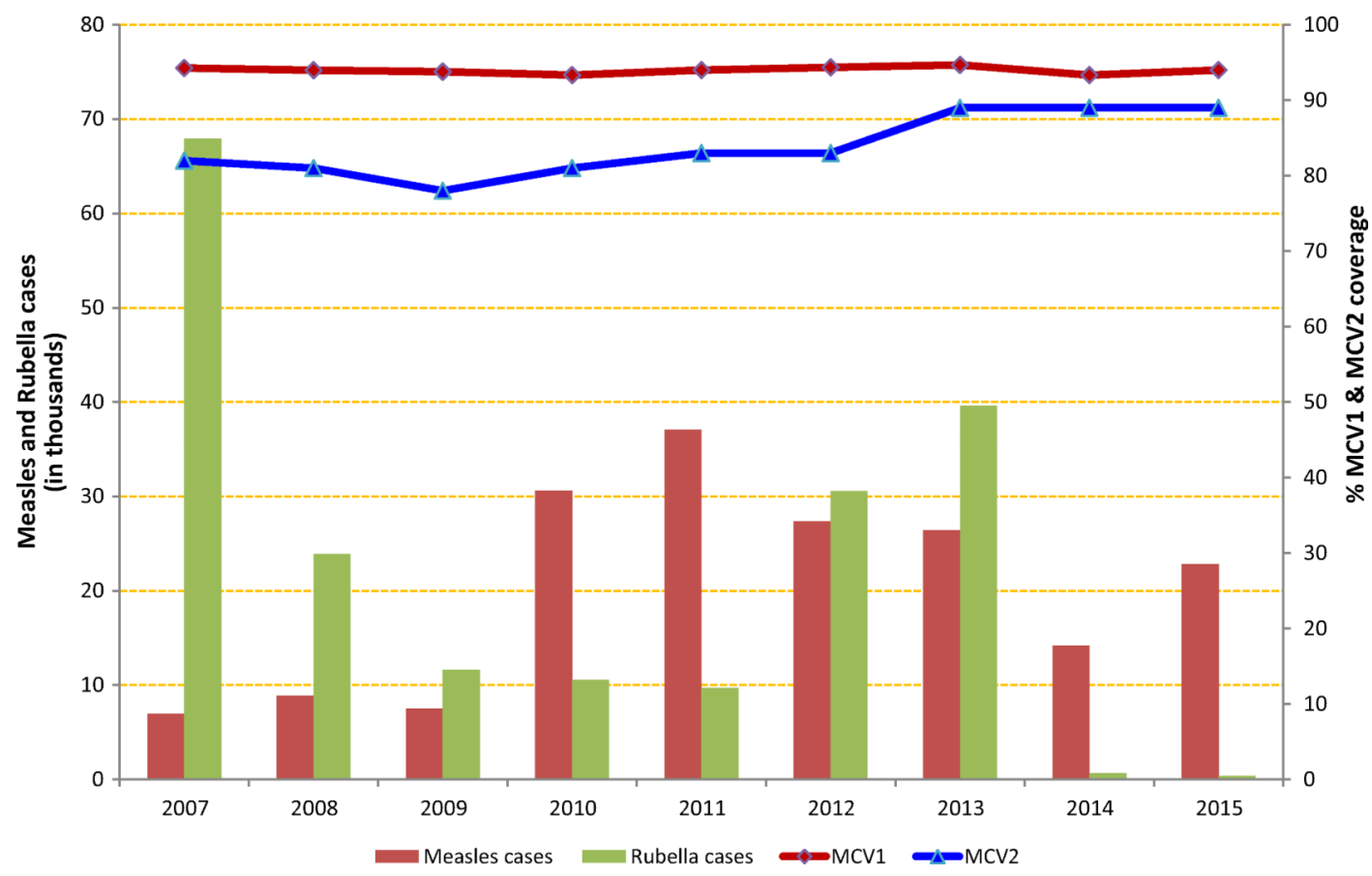

Fig. 2.

Reported measles and rubella cases, and MCV1 and MCV2 coverage by year (2007-2015) in the WHO European Region. Data source: WHO/UNICEF Joint Reporting Form data; 2007-2015 (data as of 18 January 2017). 


\section{MCV2 Coverage for 2012 and 2015, WHO European Region}

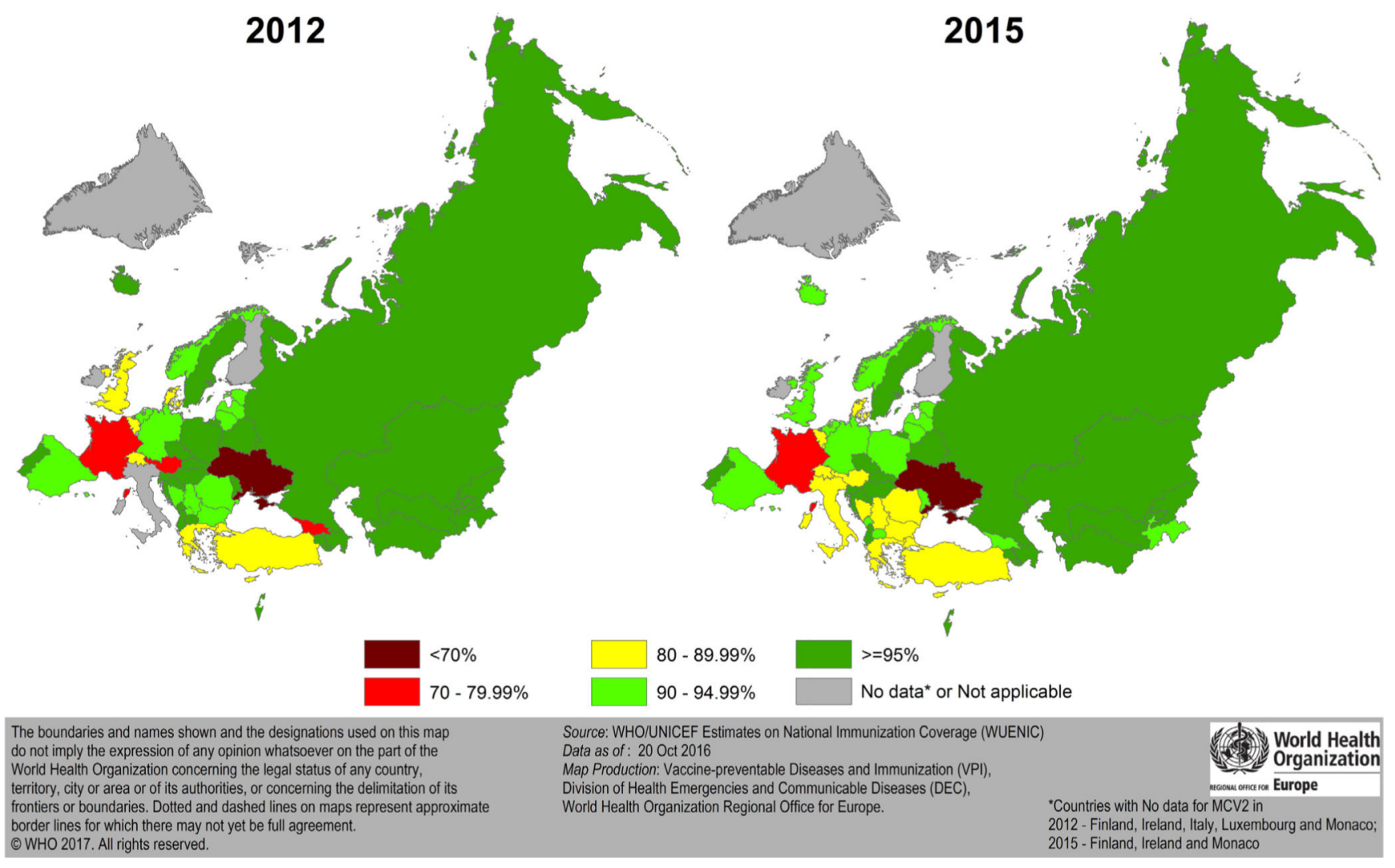

Fig. 3.

Comparison of MCV2 coverage in 2012 and 2015 in the WHO European Region. 


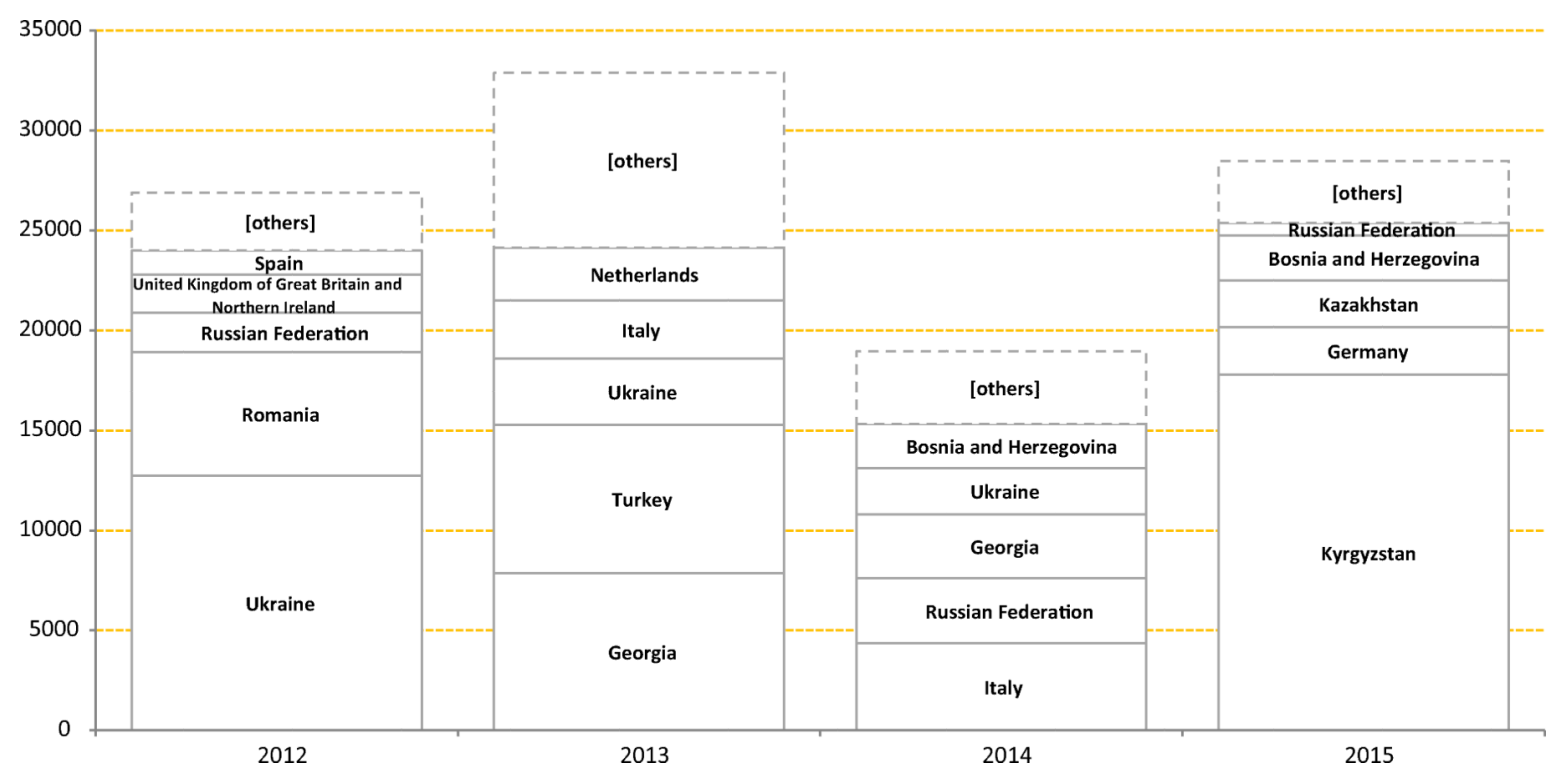

Fig. 4.

Top five countries reporting measles cases by year (2012-2015) in the WHO European Region. Data source: Centralized information system for infectious diseases; 2015 (data as of 18 January 2016). 


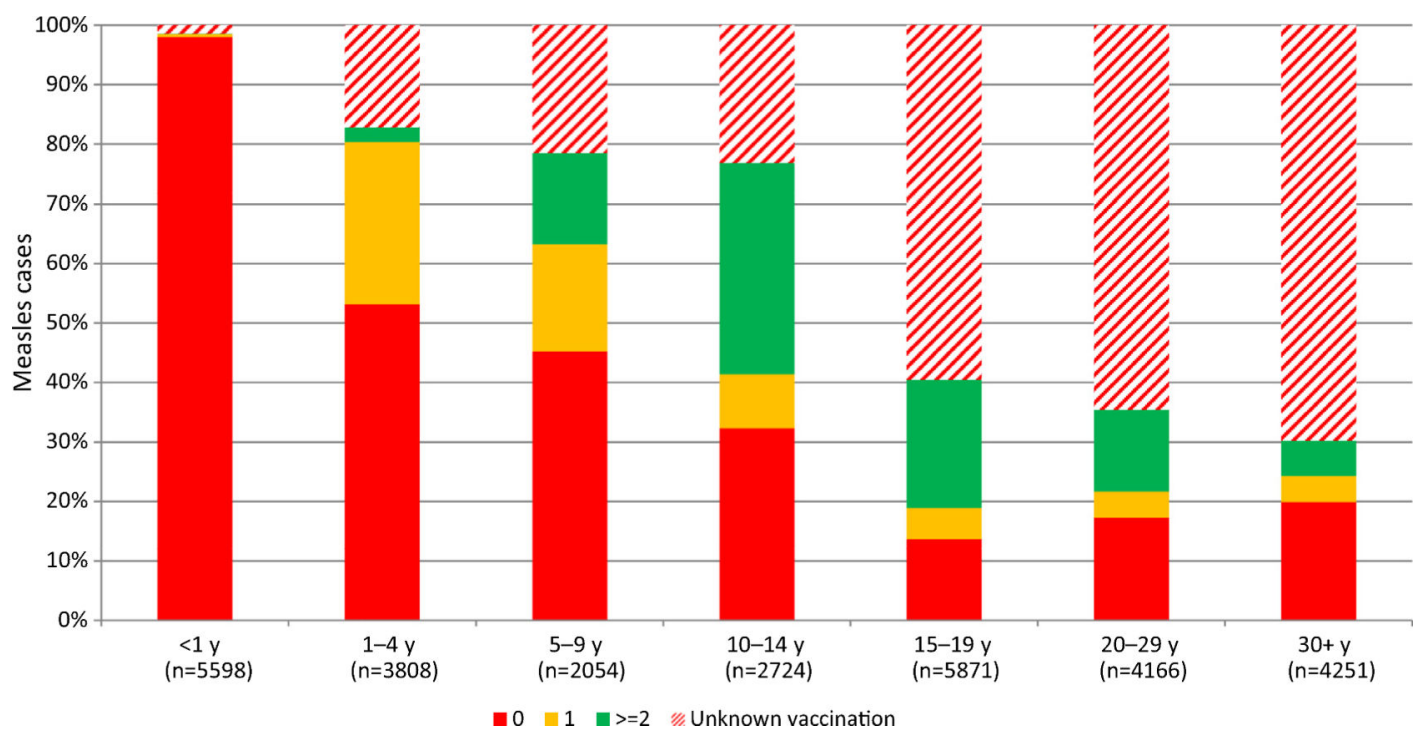

Fig. 5.

Age groups affected by measles and the vaccination status of cases in 2015 in the WHO European Region. Data source: Centralized information system for infectious diseases; 2015 (data as of 18 January 2016). 


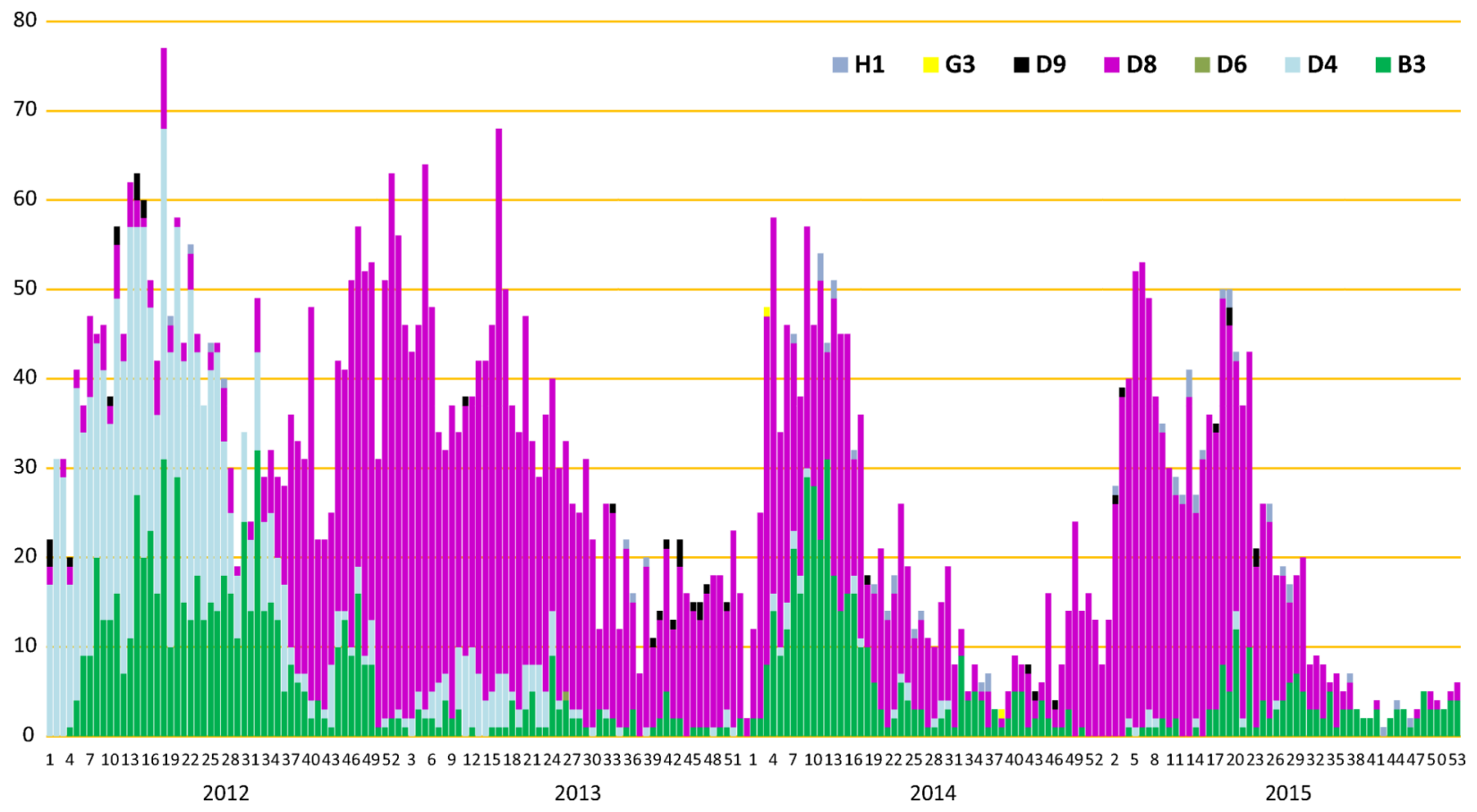

Fig. 6.

Reported measles genotype distribution by year (2012-2015) in the WHO European Region. Data source: Measles Nucleotide Surveillance database (MeaNS); 2012-2015 (data as of 18 January 2017). 